\title{
Secure Protocols with Asymmetric Trust
}

\author{
Ivan Damgård ${ }^{1}$, Yvo Desmedt ${ }^{2}$, Matthias Fitzi ${ }^{3}$, and Jesper Buus Nielsen ${ }^{1}$ \\ ${ }^{1}$ Aarhus University, Dept. of Computer Science, 8200 Aarhus N, Denmark \\ \{ivan, buus\}@daimi.au.dk \\ ${ }^{2}$ University College London, Dept. of Computer Science, London WC1E 6BT, \\ United Kingdom \\ y.desmedt@cs.ucl.ac.uk \\ ${ }^{3}$ ETH Zürich, Dept. of Computer Science, 8092 Zürich, Switzerland \\ fitzi@inf.ethz.ch
}

\begin{abstract}
In the standard general-adversary model for multi-party protocols, a global adversary structure is given, and every party must trust in this particular structure. We introduce a more general model, the asymmetric-trust model, wherein every party is allowed to trust in a different, personally customized adversary structure. We have two main contributions. First, we present non-trivial lower and upper bounds for broadcast, verifiable secret sharing, and general multi-party computation in different variations of this new model. The obtained bounds demonstrate that the new model is strictly more powerful than the standard general-adversary model. Second, we propose a framework for expressing and analyzing asymmetric trust in the usual simulation paradigm for defining security of protocols, and in particular show a general composition theorem for protocols with asymmetric trust.
\end{abstract}

\section{Introduction}

In the standard general-adversary model for multi-party computation (MPC) 13, an adversary structure is specified which basically lists all sets of parties that we expect the adversary might be able to corrupt. This model is symmetric: every party is required to trust in the same adversary structure $\mathcal{A}$. This is unnatural since there is no inherent reason why the parties should all have the same view on which adversary structure best models the given scenario. For instance, two parties may have completely contradictory beliefs on whether a third party can be corrupted or not. Also, insisting on one global adversary structure may imply that a party must consent to the fact that he himself is completely untrusted. In this paper, we introduce a more natural asymmetric-trust model where each party $p_{i}$ is allowed to trust in his own adversary structure $\mathcal{A}_{i}$. We then explore the differences between this asymmetric model and the standard one.

Of course, a trivial approach is to try to build a protocol that will be secure even if any set from any $\mathcal{A}_{i}$ is corrupt. However, this may be impossible, namely if the union of all $\mathcal{A}_{i}$ violates known lower bounds for the symmetric model. Our 
main conclusion in this paper is that there are cases where the trivial symmetric solution does not work, but where nevertheless broadcast, verifiable secret sharing, or even general secure computation, are possible with asymmetric trust.

As an example, consider the three-party scenario where $p_{1}$ distrusts $p_{2}, p_{2}$ distrusts $p_{3}$, and $p_{3}$ distrusts $p_{1}$. In the standard model, MPC requires broadcast channels for this problem. In contrast, in the most natural one of our asymmetrictrust models, MPC does not require broadcast for the same scenario.

\subsection{General Setting}

We assume that $n$ parties $\mathcal{P}=\left\{p_{1}, \ldots, p_{n}\right\}$ are given who are connected by a complete, synchronous network of pairwise channels. Also present is an adversary who may corrupt some subset of the parties.

We consider both passive and active corruption. We also consider both computational and unconditional security; where we may distinguish between unconditional security with negligible error probability or perfect security. When we do not state the type of security explicitly, positive results mean that the goal can be achieved with unconditional security, and negative results hold even w.r.t. to computational security.

A crucial point is whether the parties are additionally connected by broadcast channels and/or share a consistent public-key infrastructure (PKI). In the active case, broadcast/PKI typically allow for more resilient protocols than in the setting with only pairwise channels. Note that a PKI can be set up with respect to an unconditional pseudo-signature scheme [217]. Therefore, in the PKI setting, the achievability of a computationally secure task typically implies its feasibility with unconditional security.

\subsection{Contributions}

General multi-party computation (MPC) 2012] typically relies on the two fundamental building blocks broadcast [16] (BC, aka Byzantine agreement) and (verifiable) secret-sharing 4197] ((V)SS). It is thus interesting to know to which extent these tasks can be achieved in a certain model.

We introduce different variants of the asymmetric-trust model and corresponding definitions for broadcast, VSS, and general MPC; and give feasibility and impossibility results for these cases. Most results demonstrate that protocols for the asymmetric model are able to tolerate a strictly stronger adversary than any protocol for the symmetric model. For broadcast and VSS, we come quite close to characterizing the difference between symmetric and asymmetric trust, while the situation is much more open for general MPC.

In addition we give a general framework for augmenting security models with asymmetric trust. For concreteness we describe how to extend the UC framework [5] with asymmetric trust. This seems to be the first simulation-based security model for reasoning about asymmetric trust. Finally, we explore the issue of when UC secure MPC is possible when the parties have asymmetric trust in the setup assumptions. 


\subsection{Symmetric-Trust Model}

In the symmetric-trust model, a single adversary structure $\mathcal{A}$ is given which is a monotone subset of the power set of $\mathcal{P}, \mathcal{A} \subseteq 2^{\mathcal{P}}$. Monotone means that $A \in \mathcal{A}$ and $A^{\prime} \subset A$ imply that $A^{\prime} \in \mathcal{A}$ The goal is to achieve secure MPC for the case that an adversary corrupts the parties in exactly one set in $\mathcal{A}$. However, if the adversary manages to corrupt a set $A \notin \mathcal{A}$ then no security is guaranteed. A set $A \in \mathcal{A}$ is called maximal if there is no set $A^{\prime} \in \mathcal{A}$ that strictly contains $A$ : $\nexists A^{\prime} \in \mathcal{A}: A^{\prime} \supset A$.

The tight bounds [13] for multi-party computation in the symmetric model are summarized in the following table where the second column indicates whether broadcast channels or a public-key infrastructure (PKI) are available.

\begin{tabular}{|c|c||c|c|}
\hline STD & Broadcast/PKI & Unconditional & Computational \\
\hline \hline Passive & don`t care & $Q^{2}$ & $Q^{1}$ \\
\hline Active & available & $Q^{2}$ & $Q^{2}$ \\
\hline Active & not available & $Q^{3}$ & $Q^{3}$ \\
\hline
\end{tabular}

$$
Q^{k} \equiv\left(\forall A_{1}, \ldots, A_{k} \in \mathcal{A}: \bigcup_{i=1}^{k} A_{i} \neq \mathcal{P}\right)
$$

\subsection{Asymmetric-Trust Model}

In the asymmetric-trust model, every party $p_{i}$ has its own personalized adversary structure $\mathcal{A}_{i} \subseteq 2^{\mathcal{P}}$. We denote $\underline{\mathcal{A}}=\left(\mathcal{A}_{1}, \ldots, \mathcal{A}_{n}\right)$ as the aggregate adversary structure and define $\mathcal{A}^{*}:=\bigcup_{i=1}^{n} \mathcal{A}_{i}$. We assume that each party $p_{i}$ trusts itself, i.e., $A \in \mathcal{A}_{i} \Rightarrow p_{i} \notin A$. The set of corrupted parties is denoted by $F$.

We generally assume that all the adversary structures $\mathcal{A}_{i}$ are publicly known, so that we can use information on them in the code of our protocols. In other words, parties must make their beliefs public. Indeed, this seems necessary for our feasibility results and besides we do not believe this to be problematic: even if we were in the symmetric model and just wanted to agree on one global adversary structure, it would still seem necessary to discuss beliefs in public.

We now introduce some variants of the asymmetric-trust model. The presentation here is somewhat informal; we show later in the paper how to fully formalize it using a variant of the UC framework.

Via Symmetry. One approach is to define security for $\left(\mathcal{A}_{1}, \ldots, \mathcal{A}_{n}\right)$ via the usual symmetric notion. It is clear that if party $p_{i}$ believes that the subsets $\mathcal{A}_{i}$ could be corrupted, then $p_{i}$ would only be willing to participate in an $\mathcal{A}$ secure protocol $\pi$ if $\mathcal{A}_{i} \subseteq \mathcal{A}$ : if $\mathcal{A}_{i} \backslash \mathcal{A} \neq \emptyset$, then there exists a subset $F \subseteq$ $\left\{p_{1}, \ldots, p_{n}\right\}$ which $p_{i}$ thinks might be corrupted and which $\pi$ might not tolerate being corrupted.

${ }^{1}$ However, we allow for the loose notation of non-monotone structures $\mathcal{A}$ in which case we actually mean the structure's monotone closure, e.g., $\mathcal{A}=\left\{\left\{p_{1}\right\}\right\}$ refers to the actual structure $\mathcal{A}=\left\{\left\{p_{1}\right\}, \emptyset\right\}$. 
The definition going via symmetry insists on still giving a definition of security by specifying some subsets $F$ against which the protocol should be secure. As argued above, to allow all parties to participate in $\pi$, any such definition would have to require $\pi$ simply to tolerate the corruption structure $\mathcal{A}^{*}=\bigcup_{i=1}^{n} \mathcal{A}_{i}$. This of course gives no new views at asymmetric trust.

Via Allowed Consequences. A more interesting approach to trust is to say that in reality all subsets $F \subseteq\left\{p_{1}, \ldots, p_{n}\right\}$ can imaginably be corrupted. The party $p_{i}$ having corruption structure $\mathcal{A}_{i}$ simply means that $p_{i}$ thinks it very unlikely that a subset $A_{i} \notin \mathcal{A}_{i}$ will be corrupted. A reasonable security definition should therefore allow any corruption pattern $F \subseteq\left\{p_{1}, \ldots, p_{n}\right\}$ to occur. The goal is then (similarly to [15]) to specify for each $F \subseteq\left\{p_{1}, \ldots, p_{n}\right\}$ what consequences the corruption of $F$ is allowed to have. These consequences should ideally be such that all $p_{i}$ would be willing to participate in an $\left(\mathcal{A}_{1}, \ldots, \mathcal{A}_{n}\right)$ secure protocol.

STRICT. In the strict notion we take the standard security definitions for broadcast, VSS, and MPC, and require that no matter what subset $F \subseteq\{1, \ldots, n\}$ is corrupted, the protocol must provide full security to all uncorrupted parties. In terms of threshold security this corresponds to $t=n$ and is unattainable for most multi-party tasks. Two-party tasks like secure communication and zeroknowledge however have strictly secure implementations, possibly using setup assumptions.

Fully Relaxed. At the other extreme from strict security we consider fully relaxed security. From the set $F \subseteq\left\{p_{1}, \ldots, p_{n}\right\}$ of corrupted parties we define three types of parties: corrupted, naïve, foreseeing. A corrupted party is a party from $F$. A naïve party $p_{i}$ is honest (not from $F$ ) but it happens that $F \notin \mathcal{A}_{i}$. A foreseeing party $p_{i}$ is honest and has $F \in \mathcal{A}_{i}$. A naïve party is called naïve as it believed it very unlikely that $F$ would be corrupted, yet it was.

The fully relaxed model requires full security (in the usual sense) for the set of foreseeing parties but no security for the naïve parties. That is, a naïve party is treated like a corrupted party (although it is not controlled by the adversary).

If $\left(\mathcal{A}_{1}, \ldots, \mathcal{A}_{n}\right)=(\mathcal{A}, \ldots, \mathcal{A})$ for some common adversary structure $\mathcal{A}$, then all parties are foreseeing (and thus protected) as long as $F \in \mathcal{A}$ and all parties are naïve (and thus unprotected) as long as $F \notin \mathcal{A}$. In this sense fully relaxed security corresponds to usual $\mathcal{A}$-security.

SEMI-RELAXED. Strict security protects even naïve parties and fully relaxed security gives no security at all to naïve parties. There are different ways to define a semi-relaxed model in-between these extremes. In general, a semi-relaxed model requires full security for the set of foreseeing parties but still some partial security (to be defined) for naïve parties.

The main reason why we consider semi-relaxed models is that, in the fully relaxed model, composition of subprotocols is difficult. A naïve party may, for instance, not be able to consistently broadcast the message it wants although it 
follows the protocol. Extending some security constraints to the set of all honest parties thus allows to compose protocols more easily.

\section{Broadcast, VSS, and MPC with Asymmetric Trust}

In this section we focus on implementing broadcast, VSS, and MPC in the pointto-point model with asymmetric trust. A first observation is that in the passive case, as well as in the active case where broadcast or a PKI is given, the symmetric bounds (summarized in the table below2 still hold for any of the defined asymmetric-trust models:

Theorem 1. In the passive case and in the active case with broadcast (or PKI), broadcast, VSS, and MPC in any asymmetric model are achievable with respect to an aggregate adversary structure $\underline{\mathcal{A}}=\left(\mathcal{A}_{1}, \ldots, \mathcal{A}_{n}\right)$ if and only if they are achievable with respect to the structure $\mathcal{A}^{*}=\bigcup_{i=1}^{n} \mathcal{A}_{i}$ in the symmetric-trust model:

\begin{tabular}{|c|c|c|}
\hline & Passive & Active $(B C / P K I)$ \\
\hline Broadcast & $Q^{1}$ & $Q^{1}$ \\
\hline$(V) S S$ & $Q^{1}$ & $Q^{2}$ \\
\hline$M P C$ & $Q^{1} / Q^{2}$ & $Q^{2}$ \\
\hline
\end{tabular}

Proof. The cases where there is a protocol for any structure are trivial. For all remaining cases $Q^{2}\left(\mathcal{A}^{*}\right)$ is a tight bound in the symmetric model.

$\Leftarrow$ Trivially, if a task is achievable in the symmetric model for $\mathcal{A}^{*}$ then it is also achievable in any asymmetric model for aggregate structure $\left(\mathcal{A}^{*}, \ldots, \mathcal{A}^{*}\right)$ and thus for any $\underline{\mathcal{A}}=\left(\mathcal{A}_{1}, \ldots, \mathcal{A}_{n}\right)$ such that $\bigcup_{i=1}^{n} \mathcal{A}_{i}=\mathcal{A}^{*}$.

$\Rightarrow$ Assume any protocol in the asymmetric model for some aggregate structure $\underline{\mathcal{A}}=\left(\mathcal{A}_{1}, \ldots, \mathcal{A}_{n}\right)$ such that $\neg Q^{2}\left(\mathcal{A}^{*}\right)$. Since each party trusts itself there must be two distinct parties $p_{i}$ and $p_{j}$ and adversary sets $A_{i} \in \mathcal{A}_{i}$ and $A_{j} \in \mathcal{A}_{j}$ such that $A_{i} \cup A_{j}=\mathcal{P}$, and $p_{i} \in A_{j}$ and $p_{j} \in A_{i}$. From this, we can build a two-party protocol for the same task wherein the parties distrust each other. This is done by having one party simulate $p_{i}$ (and the parties in $A_{j}$ ) and the other one $p_{j}$ (and the parties in $A_{i}$ ), and then execute the asymmetric protocol we assumed exists.

For unconditionally secure MPC in the passive case this implies that two parties can securely compute the logical OR over their input bits, which is impossible 318.

For VSS (in the active case) this implies that a dealer can secret-share a value in the two-party setting such that the other party can reconstruct it during the reconstruction phase without the help of the dealer - but then it can also do so at any time after the sharing phase, which contradicts security.

${ }^{2}$ The only difference between computational and unconditional security occurs for MPC in the passive case where MPC for any structure is achievable with computational security but $Q^{2}$ is necessary for unconditional security. 
For MPC (and secure function evaluation, in particular) in the active case this implies that two parties can flip a fair coin, which is impossible [8].

In view of this theorem, for the rest of this section, we concentrate on the case where the adversary is active and where BC/PKI are not assumed.

\subsection{Broadcast}

Definition 1 (Broadcast $\mathrm{w} /$ full relaxation). A protocol where sender $p_{s} \in$ $\mathcal{P}$ inputs $x_{s} \in \mathcal{D}$, and all $p_{i} \in \mathcal{P}$ output $y_{i} \in \mathcal{D}$, achieves broadcast with full relaxation if:

VALIDITY: If $p_{s}$ and $p_{i}$ are honest and $F \in \mathcal{A}_{s} \cap \mathcal{A}_{i}$ then $y_{i}=x_{s}$.

Consistency: If $p_{i}$ and $p_{j}$ are honest and $F \in \mathcal{A}_{i} \cap \mathcal{A}_{j}$ then $y_{i}=y_{j}$.

For the use as a subprotocol in MPC, a helpful additional property of broadcast is to demand validity independently of the sender's adversary structure. In that way, a naïve party can still consistently convey its view. We define semi-relaxed broadcast as broadcast with sender-independent validity in the following way where we only state the different validity condition.

\section{Definition 2 (Broadcast $\mathrm{w} /$ sender-indep. validity (semi-relaxed)).}

VALIDITY: If $p_{s}$ and $p_{i}$ are honest and $F \in \mathcal{A}_{i}$ then $y_{i}=x_{s}$.

The following theorem is proven in the full version of the paper.

Theorem 2. Broadcast with sender-independent validity for every sender $p_{s} \in$ $\mathcal{P}$ is (perfectly) achievable if and only if

$$
B^{3}(\underline{\mathcal{A}}) \equiv \forall \mathcal{A}_{i}, \mathcal{A}_{j}: \forall A_{i} \in \mathcal{A}_{i}, A_{j} \in \mathcal{A}_{j}, A_{i j} \in \mathcal{A}_{i} \cap \mathcal{A}_{j}: A_{i} \cup A_{j} \cup A_{i j} \neq \mathcal{P} .
$$

Note that $B^{3}(\underline{\mathcal{A}})$ is a proper relaxation of $Q^{3}(\underline{\mathcal{A}})$, which is necessary and sufficient in the symmetric framework. In particular, $B^{3}(\underline{\mathcal{A}})$ is a condition on all pairs of parties, whereas $Q^{3}(\underline{\mathcal{A}})$ is the condition $\forall A_{i} \in \mathcal{A}_{i}, \forall A_{j} \in \mathcal{A}_{j}, \forall A_{k} \in \mathcal{A}_{k}$ : $A_{i} \cup A_{j} \cup A_{k} \neq \mathcal{P}$ on all triples of parties. Trivially, any semi-relaxed version of broadcast implies broadcast with full relaxation. Achievability under $B^{3}(\underline{\mathcal{A}})$ thus follows for the fully relaxed case. However, the next two results show, first that $B^{3}(\underline{\mathcal{A}})$ is not necessary for fully relaxed broadcast, and second, a weaker but necessary condition.

Proposition 1. There are aggregate structures $\underline{\mathcal{A}}$ such that $\neg B^{3}(\underline{\mathcal{A}})$ and broadcast with full relaxation is achievable for every selection of a sender $p_{s} \in \mathcal{P}$.

Proof. Consider aggregate structure $\underline{\mathcal{A}}=\left(\left\{\left\{p_{2}\right\},\left\{p_{3}\right\}\right\},\left\{\left\{p_{1}\right\},\left\{p_{3}\right\}\right\}, \emptyset\right)$ among $\mathcal{P}=\left\{p_{1}, p_{2}, p_{3}\right\}$. If the sender is $p_{1}$ or $p_{2}$ then it can simply multi-send its input value since, with respect to $p_{3}$, validity and consistency only have to hold if nobody is corrupted. If the sender is $p_{3}$ then $p_{3}$ can send its input value to $p_{1}$ who in turn sends it to $p_{2}$. Again, validity and consistency with respect to $p_{3}$ only have to hold if no party is corrupted; parties $p_{1}$ and $p_{2}$ are trivially consistent. 
Theorem 3. If there are structures $\mathcal{A}_{i}, \mathcal{A}_{j}$, and $\mathcal{A}_{k}$, and sets $A_{i j} \in \mathcal{A}_{i} \cap \mathcal{A}_{j}$, $A_{i k} \in \mathcal{A}_{i} \cap \mathcal{A}_{k}, A_{j k} \in \mathcal{A}_{j} \cap \mathcal{A}_{k}$, such that $A_{i j} \cup A_{i k} \cup A_{j k}=\mathcal{P}$ then broadcast with full relaxation is not achievable for any selection of a sender $p_{s} \in \mathcal{P}$.

Proof. Along the lines of the lower-bound part of the proof of Theorem 2 .

\subsection{Verifiable Secret Sharing (VSS)}

Definition 3 (VSS w/ full relaxation). A pair of protocols (Sh, Rec) wherein dealer $p_{d} \in \mathcal{P}$ inputs secret $s$ in protocol Sh and every $p_{i} \in \mathcal{P}$ outputs $s_{i}$ in protocol Rec achieves VSS with full relaxation if:

SECRECY: If $p_{d}$ is honest and $F \in \mathcal{A}_{d}$ then the adversary has no information about $s$ as long as protocol Rec has not started yet.

Correctness: If $p_{d}$ and $p_{i}$ are honest and $F \in \mathcal{A}_{d} \cap \mathcal{A}_{i}$ then $p_{i}$ computes output $s_{i}=s$ in protocol Rec.

Commitment: If $p_{i}$ and $p_{j}$ (case $i=j$ included) are honest and $F \in \mathcal{A}_{i} \cap \mathcal{A}_{j}$ then, after termination of protocol $\mathrm{Sh}$, there is a value $s^{\prime} \in \mathbb{F}$ such that, in protocol Rec, $p_{i}$ and $p_{j}$ compute output $s_{i}=s_{j}=s^{\prime}$.

It may be tempting to believe that fully relaxed VSS could be obtained by just running a standard VSS protocol that is secure with respect to the dealer's adversary structure $\mathcal{A}_{d}$. But such a protocol provides no security at all if $F \notin \mathcal{A}_{d}$, and hence cannot in general guarantee that the commitment property is satisfied.

We define semi-relaxed VSS as VSS with dealer-independent correctness in the following way - where we only state the conditions different from the previous definition.

Definition 4 (VSS w/ dealer-indep. correctness (semi-relaxed)).

Correctness: If $p_{d}$ and $p_{i}$ are honest and $F \in \mathcal{A}_{i}$ then $p_{i}$ computes output $s_{i}=s$ in protocol Rec.

We derive our VSS protocols from the VSS protocol in 14. Note that, since we are not given full-fledged broadcast, additional measures have to be taken.

Theorem 4. Perfectly secure VSS with full relaxation is achievable for every selection of a dealer $p_{d} \in \mathcal{P}$ if

$$
V^{3}(\underline{\mathcal{A}}) \equiv \forall \mathcal{A}_{i}, \mathcal{A}_{j}: \forall A_{i} \in \mathcal{A}_{i}, \forall A_{i}^{\prime} \in \mathcal{A}_{i}, \forall A_{j} \in \mathcal{A}_{j}: \quad A_{i} \cup A_{i}^{\prime} \cup A_{j} \neq \mathcal{P} .
$$

Proof. Follows from the protocol in Fig. 1 which is analyzed in Lemma 1 .

Lemma 1. For a given $V^{3}$-structure, the protocol in Fig. 1 achieves fully relaxed VSS with perfect security.

Proof. Secrecy: If $F \in \mathcal{A}_{d}$ then the share $s_{k}$ with $P_{k}=\mathcal{P} \backslash F \neq \emptyset$ being all honest does not get opened during the sharing phase by an honest dealer since it receives no complaints from within $P_{k}$ with respect to this share (all broadcasts are valid with respect to $p_{d}$ ). Share $s_{k}$ perfectly hides the secret. 
Sharing Sh: For each maximal set $A_{k} \in \mathcal{A}_{d}$ the dealer $p_{d}$ assigns a random share $s_{k} \in \mathbb{F}$ with the only restriction that $s=\sum_{k} s_{k}$. The dealer sends each $s_{k}$ to all parties in $P_{k}=\mathcal{P} \backslash A_{k}$. Each party $p_{i} \in P_{k}$ stores $s_{k}$ and $s_{k}^{\prime}:=s_{k}$.

For each $s_{k}$, the parties in $P_{k}$ pairwisely compare their shares. If any inconsistency is detected by a party $p_{i} \in P_{k}$ it broadcasts (w/ sender-independent validity) a complaint to all parties in $\mathcal{P}$.

Now, if the dealer receives any complaint, it opens share $s_{k}$ by broadcast (w/ senderindependent validity) towards $\mathcal{P}$. Party $p_{i} \in \mathcal{P}$ always adopts any opening by the dealer. If a party $p_{i} \in \mathcal{P}$ sent or received a complaint but does not see the dealer open $s_{k}$, it disqualifies the dealer and defines $s_{k}:=0$. Note that a party in $P_{k}$ who disqualifies the dealer still stores the initial share $s_{k}^{\prime}$ it is holding - although, from now on, it uses $s_{k}=0$ for its own computation.

A party $p_{i} \in A_{k}$ who, at this point, neither disqualified the dealer nor saw the dealer open share $s_{k}$, is called $k$-curious.

Reconstruction Rec: For each share $s_{k}$,

- All parties in $P_{k}$ multi-send $s_{k}^{\prime}$ to the parties in $A_{k}$.

- All parties who are not $k$-curious accept $s_{k}$ as the reconstructed share.

- All parties $p_{j}$ who are $k$-curious wait for the parties $p_{i} \in P_{k}$ to send their shares. Then they search for a set $A_{j} \in \mathcal{A}_{j}$ such that all parties in $P_{k} \backslash A_{j}$ sent the same share $\hat{s}_{k}^{\prime}$. Party $p_{j}$ then accepts $s_{k}:=\hat{s}_{k}^{\prime}$.

Finally, all shares $s_{k}$ are summed up in order to compute the reconstructed secret.

Fig. 1. Protocol VSS with full relaxation

Correctness: We show that when parties $p_{d}$ and $p_{i}$ are honest and $F \in$ $\mathcal{A}_{d} \cap \mathcal{A}_{i}$ then, during reconstruction, $p_{i}$ opens each share $s_{k}$ (share with respect to $A_{k} \in \mathcal{A}$ ) correctly as distributed by $p_{d}$.

First, we observe that $p_{i}$ does not disqualify the dealer $p_{d}$ : disqualification implies a complaint sent or received by $p_{i}$ - and thus also received by $p_{d}$. This forces $p_{d}$ to open $s_{k}$, implying that $p_{i}$ does not disqualify $p_{d}$. This implies that either $p_{d}$ opened $s_{k}$ during the sharing phase or that all honest parties in $P_{k}$ agree on the same share $s_{k}^{\prime}=s_{k}$. An opening during the sharing phase is correctly received by $p_{i}$ (validity of broadcast). If $p_{i}$ remains $k$ curious then there is the unique value $\hat{s}_{k}^{\prime}=s_{k}$ such that there exists some $A_{i} \in \mathcal{A}_{i}$ with all parties in $P_{k} \backslash A_{i}$ opening the same share $\hat{s}_{k}^{\prime}$ - since $A_{d} \cup A_{i} \cup A_{i}^{\prime} \neq \mathcal{P}$.

Commitment: Consider two honest parties $p_{i}$ and $p_{j}$ such that $F \in \mathcal{A}_{i} \cap \mathcal{A}_{j}$. All information that is broadcast is thus valid and consistent with respect to $p_{i}$ and $p_{j}$. We distinguish three cases.

$-p_{i}, p_{j} \in P_{k}$. Because of broadcast consistency, both parties either disqualify the dealer $\left(s_{k}=0\right)$, or accept the same initial share, or adopt the same share being opened by the dealer.

- $p_{i} \in P_{k}, p_{j} \in A_{k}$. Because of broadcast consistency, $p_{i}$ and $p_{j}$ receive exactly the same values that are broadcast. Thus either both disqualify, or both adopt, or $p_{i}$ stays with his initial share whereas $p_{j}$ is $k$-curious. In the latter case, there was no complaint and thus no conflict among any honest parties in $P_{k}$ - and thus all honest parties in $P_{k}$ hold the 
same share $s_{k}^{\prime}$. As in the correctness argument, $p_{j}$ thus finds the unique value $\hat{s}_{k}^{\prime}=s_{k}$ such that there is some $A_{j} \in \mathcal{A}_{j}$ with all parties in $P_{k} \backslash A_{j}$ all opening the same share $\hat{s}_{k}^{\prime}$ — which is identical to $p_{i}$ 's share. Thus, commitment is also guaranteed in the latter case.

- $p_{i}, p_{j} \in A_{k}$. The parties both either are $k$-curious, or adopt the same opening, or disqualify the dealer. If they are $k$-curious then no honest party in $P_{k}$ broadcast a complaint and thus, again, all honest parties in $P_{k}$ hold the same share $s_{k}^{\prime}$. Since $F \in \mathcal{A}_{i} \cap \mathcal{A}_{j}$ both parties will determine a set $A_{i} \subseteq F$ (and $A_{j} \subseteq F$, respectively) such that the parties in $P_{k} \backslash A_{i}$ $\left(P_{k} \backslash A_{j}\right)$ all open the same share $\hat{s}_{k}^{\prime}=s_{k}^{\prime}$.

Proposition 2. There are aggregate structures $\underline{\mathcal{A}}$ such that $\neg V^{3}(\underline{\mathcal{A}})$ and $V S S$ with full relaxation is achievable for every selection of a dealer $p_{d} \in \mathcal{P}$.

Proof. Consider aggregate structure $\underline{\mathcal{A}}=\left(\left\{\left\{p_{2}\right\},\left\{p_{3}\right\}\right\},\left\{\left\{p_{1}\right\}\right\}, \emptyset\right)$ among $\mathcal{P}=$ $\left\{p_{1}, p_{2}, p_{3}\right\}$. The parties can run the preprocessing protocol from [10] trying to establish a PKI with unconditional security. If it succeeds then the players can simulate broadcast and thus use the VSS protocol for dishonest minorities in, e.g., 9]. If it fails then it suffices that the dealer always reconstructs his input value whereas the other parties reconstruct some default value.

The following theorem is proven in the full version of the paper.

Theorem 5. Unconditionally secure VSS with dealer-independent correctness is achievable if $V^{3}(\underline{\mathcal{A}})$. Additionally, secrecy with respect to any $F \in \mathcal{A}^{*}$ can be guaranteed.

Theorem 6. If $\neg V^{3}(\underline{\mathcal{A}})$ then perfectly secure VSS with dealer-independent correctness is not achievable for every selection of a dealer $p_{d} \in \mathcal{P}$.

Proof. If $n=2$ then $\neg V^{3}(\underline{\mathcal{A}})$ and self-trust imply $\neg Q^{2}\left(\mathcal{A}^{*}\right)$, and impossibility follows from Theorem 1. We can therefore assume that $n \geq 3$.

With $\neg V^{3}(\underline{\mathcal{A}})$ there are structures $\mathcal{A}_{i}$ and $\mathcal{A}_{j}$, and sets $A_{i}, A_{i}^{\prime} \in \mathcal{A}_{i}$ and $A_{j} \in \mathcal{A}_{j}$ with $A_{i} \cup A_{i}^{\prime} \cup A_{j}=\mathcal{P}$. We show that there is no VSS with respect to dealer $p_{d}=p_{j}$. Note that $A_{d} \cup A_{i} \cup A_{i}^{\prime}=\mathcal{P}$ and self-trust imply, wlog, that $p_{d} \in A_{i}$ and $p_{i} \in A_{d}$.

If such a VSS protocol existed then three parties $p_{\delta}, p_{\iota}$ and $p_{\kappa}$ could use it to simulate VSS among themselves with dealer $p_{\delta}$ where $\left(\mathcal{A}_{\delta}, \mathcal{A}_{\iota}, \mathcal{A}_{\kappa}\right)=$ $\left\{\left\{p_{\iota}\right\},\left\{\left\{p_{\delta}\right\},\left\{p_{\kappa}\right\}\right\}, \emptyset\right\}: p_{\delta}$ simulates all parties in $A_{i}, p_{\iota}$ simulates all parties in $A_{d}$, and $p_{\kappa}$ simulates all parties in $A_{i}^{\prime}$. Now the share $s_{\iota}$ is not allowed to give any information about secret $s$ but any triplet $\left(s_{\delta}, s_{\iota}, \cdot\right)$ perfectly reveals an honest dealer's correct secret and any triplet $\left(\cdot, s_{\iota}, s_{\kappa}\right)$ perfectly reveals the value a corrupted dealer was committed to. This is not possible.

Finally, note that impossibility of broadcast implies impossibility of VSS. Thus all impossibility results for broadcast naturally extend to VSS. 


\subsection{Multi-Party Computation (MPC)}

We now argue informally that, also with respect to general MPC, the asymmetric-trust model allows to tolerate strictly more than in the symmetric case. We only consider fully relaxed security, i.e., privacy and correctness only hold for foreseeing parties. This notion of MPC security is formalized in the following section.

Theorem 7. In the fully relaxed model, there exist infinite many aggregate structures $\underline{\mathcal{A}}=\left(\mathcal{A}_{1}, \ldots, \mathcal{A}_{n}\right)$ with $\neg Q^{3}\left(\mathcal{A}^{*}\right)$ for which unconditionally secure $M P C$ is achievable.

Proof. We construct an aggregate adversary structure $\mathcal{A}$ for $n=3 T$ parties, where each individual $\mathcal{A}_{i}$ is such that its maximal sets have size $T$, but where no set of size $T$ occurs in more than one $\mathcal{A}_{i}$. Clearly, for each $n$, there are several such structures and several of them are not $Q^{3}$. For such a structure, we can implement MPC by first running a preprocessing protocol from [11 that aims at establishing a PKI with unconditional security (as discussed earlier). This protocol in its most general form has parameters $T$ and $t$, where $2 T+t<n$; we choose $t=T-1$. The protocol guarantees success if there are at most $t$ corruptions. If there are at most $T$, there will be agreement on the result which is "success" or "failure." Our solution is that, if the preprocessing is successful, we run a standard MPC protocol secure against $T$ corruptions based on the PKI constructed. If the preprocessing fails, each party computes its output locally using its own input and default values for the other parties. As for security, note first that if there are more than $T$ corruptions, all parties are naïve or corrupted, and security is guaranteed. If there are at most $T-1=t$ corruptions, the preprocessing succeeds, and the protocol is secure. If there are $T$ corruptions, either the preprocessing succeeds, in which case we are fine, as before. Otherwise, all honest parties agree that it failed. Since the corrupted set occurs in at most one of the $\mathcal{A}_{i}$, at most one party is foreseeing, and it may securely compute its output locally since the fully relaxed requirement only forces foreseeing parties to be consistent. All other parties are naïve or corrupt.

Again, the impossibility results for broadcast naturally extend to MPC.

\section{A Generic Framework for Asymmetric Trust}

Until now we gave ad-hoc definitions of asymmetric security for VSS and broadcast. We now develop a general framework for augmenting security models with asymmetric trust. The asymmetric security notions introduced above can be derived as special cases. The exposition is meant as a framework for adding asymmetric trust to protocol security models phrased via ideal functionalities and corruptions. For concreteness we consider the UC framework. 


\subsection{Basic UC Framework}

We consider protocols $\pi$ for a party set $\mathcal{P}=\left\{p_{1}, \ldots, p_{n}\right\}$. A corruption pattern is a pair of subsets PAT $=(\mathrm{ACT}, \mathrm{PAS}), \mathrm{ACT}, \mathrm{PAS} \subseteq \mathcal{P}$, where $\mathrm{ACT} \subseteq \mathrm{PAS}$; The interpretation is that the parties $p_{i} \in$ PAS are passively corrupted and the parties $p_{i} \in \mathrm{ACT}$ actively corrupted. We write $(\mathrm{ACT}, \mathrm{PAS}) \subseteq\left(\mathrm{ACT}^{\prime}, \mathrm{PAS}^{\prime}\right)$ to mean $\mathrm{ACT} \subseteq \mathrm{ACT}^{\prime}$ and PAS $\subseteq \mathrm{PAS}^{\prime}$. An adversary structure is a set $\mathcal{A}=\{(\mathrm{ACT}, \mathrm{PAS})\}$ of corruption patterns, where $\mathrm{PAT} \in \mathcal{A} \wedge \mathrm{PAT}^{\prime} \subseteq \mathrm{PAT} \Rightarrow \mathrm{PAT}^{\prime} \in \mathcal{A}$.

An ideal functionality is an ITM $\mathcal{F}$. It can receive inputs from each $p_{i} \in \mathcal{P}$ ( $p_{i}$-inputs) and deliver outputs to each $p_{i} \in \mathcal{P}$ ( $p_{i}$-outputs). Besides this, it can receive aux-inputs and deliver aux-outputs, thought of as inputs coming from the adversary respectively values leaked to the adversary. As an example an ideal functionality $\mathcal{F}_{\text {Com }}$ for bit commitment can be phrased as follows: On $p_{i^{-}}$ input (commit, cid, $p_{i}, p_{j}, m \in\{0,1\}$ ) produce aux-output (commit, cid, $p_{i}, p_{j}$ ); Here $c i d$ is a commitment identifier. On a later aux-input (deliver, $c i d, p_{i}, p_{j}$ ), output (receipt, $c i d, p_{i}, p_{j}$ ) to $p_{j}$. On $p_{i}$-input (open, $c i d, p_{i}, p_{j}$ ) after receiving $p_{i}$-input (commit, $\left.c i d, p_{i}, p_{j}, m\right)$, produce aux-output (open, $c i d, p_{i}, p_{j}, m$ ). On a later aux-input (open, $c i d, p_{i}, p_{j}$ ), output (open, $c i d, p_{i}, p_{j}, m$ ) to $p_{j}$.

A protocol $\pi$ consists of $n$ parties $p_{1}, \ldots, p_{n}$ and some ideal functionalities $\mathcal{G}$, which might, e.g., model point-to-point lines or commitment. We write $\mathcal{G} \in \pi$ and $\pi[\mathcal{G}]$ to mean that $\pi$ uses the ideal functionality $\mathcal{G}$. An environment $\mathcal{Z}$ for $\pi$ is a ITM which gives inputs to the parties and gets outputs from the parties. We denote an execution of $\pi$ in $\mathcal{Z}$ by $\operatorname{ExEC}_{\pi, \mathcal{Z}}$. The environment $\mathcal{Z}$ also corrupts parties 3 For a corruption pattern $\mathrm{PAT}=(\mathrm{ACT}, \mathrm{PAS})$ the environment is allowed to see the internal state of $p_{i} \in$ PAS and control $p_{i} \in$ ACT: When $p_{i} \in$ ACT, then in $\operatorname{ExEC}_{\pi, \mathcal{Z}}$ it is $\mathcal{Z}$ which determines all $p_{i}$-inputs to $\mathcal{G} \in \pi$ and receives all $p_{i}$-outputs from $\mathcal{G} \in \pi$. The party $p_{i}$ is not run at all. Besides this, $\mathcal{Z}$ receives all aux-outputs from all $\mathcal{G} \in \pi$ and can give aux-inputs to all $\mathcal{G} \in \pi$. As an example, in $\operatorname{ExEC}_{\pi\left[\mathcal{F}_{\mathrm{Com}}\right], \mathcal{Z}}$ the environment sees when commitments are made and determines when to deliver receipts and openings.

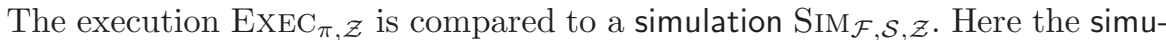
lator $\mathcal{S}$ must simulate an execution of $\pi$. E.g., $\mathcal{S}$ simulates aux-outputs to $\mathcal{Z}$ from all $\mathcal{G} \in \pi$ and receives aux-inputs from $\mathcal{Z}$ to $\mathcal{G} \in \pi$. The simulator itself receives aux-outputs from $\mathcal{F}$ and gives aux-inputs to $\mathcal{F}$. When $\mathcal{Z}$ gives a $p_{i}$-input for $p_{i} \notin \mathrm{ACT}$, it is given to $\mathcal{F}$. The simulator gives all $p_{i}$-inputs to $\mathcal{F}$ for $p_{i} \in \mathrm{ACT}$. When $\mathcal{F}$ produces a $p_{i}$-output for $p_{i} \notin \mathrm{ACT}$, it is given to $\mathcal{Z}$, but when $\mathcal{F}$ produces a $p_{i}$-output for $p_{i} \in \mathrm{ACT}$, it is not given to $\mathcal{Z}$. When $\mathcal{Z}$ gives a $p_{i}$-input to $\mathcal{F}$ for $p_{i} \in$ PAS, it is shown to $\mathcal{S}$, and when $\mathcal{F}$ produces a $p_{i}$-output for $p_{i} \in$ PAS, it is shown to $\mathcal{S}$.

A protocol $\pi$ is called a UC secure implementation of $\mathcal{F}$ if there exists a simulator $\mathcal{S}$ such that $\operatorname{Sim}_{\mathcal{F}, \mathcal{S}, \mathcal{Z}} \approx \operatorname{ExEC}_{\pi, \mathcal{Z}}$ for all $\mathcal{Z}$. It is possible to restrict $\mathcal{Z}$ to corrupting according to some $\mathrm{PAT} \in \mathcal{A}$, in which case we say that $\pi$ is $\mathcal{A}$-secure (in the symmetric sense).

${ }^{3}$ We use the formulation of the UC framework without an explicit adversary, see full version of [5]. 
$\mathcal{F}^{\text {(Xtn) }}$ runs a copy of $\mathcal{F}$. When $\mathcal{F}$ produces aux-output $z, \mathcal{F}^{(\mathrm{Xtn})}$ produces aux-output $z$, and when $\mathcal{F}^{(\mathrm{Xtn})}$ receives aux-input $z$ it gives $\mathcal{F}$ the auxinput $z$. When the (current) corruption pattern is PAT and $\mathrm{Xtn}(\mathrm{PAT})=$ (ActIn, ActOut, PasIn, PAsOut), the remaining inputs and outputs are handled as follows:

- For $p_{i} \in$ PASIN: On $p_{i}$-input $x$, produce aux-output (in, $p_{i}, x$ ), and then give $\mathcal{F}$ the $p_{i}$-input $x$.

- For $p_{i} \in$ PASOut: On $p_{i}$-output $x$ from $\mathcal{F}$, produce aux-output (out, $p_{i}, x$ ), and then produce the $p_{i}$-output $x$.

- For $p_{i} \in$ ACTIn: Ignore all $p_{i}$-inputs, and on an aux-input (in, $\left.p_{i}, x\right)$, give $\mathcal{F}$ the $p_{i}$-input $x$.

- For $p_{i} \in$ ACTOUT: Ignore all $p_{i}$-outputs from $\mathcal{F}$, and on an aux-input (out, $\left.p_{i}, x\right)$, produce the $p_{i}$-output $x$.

Fig. 2. $\mathcal{F}^{(\mathrm{Xtn})}$

\subsection{Modeling the Security Loss of Naïve Parties}

To define asymmetric trust in the UC framework, we need to model the loss of security we will allow for a party who turns out to have been naïve. To express what we choose to allow, we introduce the concept of a corruption extension Xtn which is a function that maps a corruption pattern PAT to a tuple

$$
\operatorname{Xtn}(\text { Pat })=(\text { Actin, ActOut, PasIn, PasOut }),
$$

of party subsets, where

\section{Pasin, PasOut $\subseteq \mathcal{P} \backslash$ Pas and Actin, ActOut $\subseteq \mathcal{P} \backslash$ Act}

These are subsets of parties who are not corrupt but nevertheless have their security violated in some way.

This is modeled in the simulation $\operatorname{Sim}_{\mathcal{F}, \mathcal{S}, \mathcal{Z}}$ by giving $\mathcal{S}$ the following extra power over $\mathcal{F}$ : For the parties $p_{i} \in$ PAsIn, respectively $p_{i} \in$ PAsOut, we show $\mathcal{S}$ the $p_{i}$-inputs to $\mathcal{F}$, respectively the $p_{i}$-outputs from $\mathcal{F}$. For the parties $p_{i} \in$ ACTIN, when $\mathcal{Z}$ gives a $p_{i}$-input to $\mathcal{F}$, it is not given to $\mathcal{F}$. Instead we allow $\mathcal{S}$ to specify these $p_{i}$-inputs. Finally, for the parties $p_{i} \in$ ACTOUT, when $\mathcal{F}$ produces a $p_{i}$-output to $\mathcal{Z}$, it is not given to $\mathcal{Z}$ but we allow $\mathcal{S}$ to specify these $p_{i}$-outputs.

Of course, a functionality $\mathcal{F}$ may also be used as an auxiliary functionality in a protocol. In this case the extra power is given to the environment (adversary), see more on this below.

In order to formally incorporate the above into the UC framework without making changes that require us to reprove the composition theorem, we define the following way to extend any ideal functionality: For a functionality $\mathcal{F}$ and any extension Xtn, let $\mathcal{F}^{(\mathrm{Xtn})}$ be the ideal functionality in Fig. 2. We say that $\pi$ is an Xtn-secure implementation of $\mathcal{F}$ if $\pi$ is a UC secure implementation of $\mathcal{F}^{\text {(Xtn) }}$ (tolerating environments corrupting any subset of parties). 
Note that in $\operatorname{SIM}_{\mathcal{F}(\mathrm{Xtn}), \mathcal{Z}}$ it is $\mathcal{S}$ which has access to the aux-inputs and auxoutputs of $\mathcal{F}^{(\mathrm{Xtn})}$, giving it exactly the desired extra power. Note also that $\mathcal{F}^{(\mathrm{Xtn})}$ is allowed in the UC framework since it allows functionalities to know which parties are corrupted.

Note that in the above definition we do not restrict in any way how many parties the environment corrupts! We might however use Xtn to specify that for some corruptions $A \notin \mathcal{A}$ the simulator is allowed to corrupt all parties in the simulation. This allows to model that for corruptions $A \notin \mathcal{A}$ no security guarantees are given. The notion of corruption extensions therefore subsumes the normal notion of restricting the environment to certain corruption patterns $\mathcal{A}$.

As mentioned, extensions also apply to a functionality $\mathcal{G}$ used in protocol $\pi$. For this purpose we assume that $\pi$ associates to each $\mathcal{G} \in \pi$ an extension $\mathrm{Xtn}_{\mathcal{G}}$. We then let $\widehat{\pi}$ denote the protocol where each $\mathcal{G} \in \pi$ is replaced by $\mathcal{G}^{(\mathrm{Xtn}} \mathrm{G}_{\mathcal{G}}$. In $\operatorname{ExEC}_{\widehat{\pi}, \mathcal{Z}}$ it is $\mathcal{Z}$ which has access to the aux-inputs and outputs of $\left.\mathcal{G}^{(\mathrm{Xtn}}{ }_{\mathcal{G}}\right)$, granting it extra power over $\mathcal{G}$ in the same way as we did for the simulator before.

Definition 5. Let $\pi$ be a protocol having an extension $\mathrm{Xtn}_{\mathcal{G}}$ associated to each $\mathcal{G} \in \pi$, let $\mathrm{Xtn}$ be some extension and let $\mathcal{F}$ be some ideal functionality. We say that $\pi$ is an Xtn-secure implementation of $\mathcal{F}$ if $\widehat{\pi}$ is a UC secure implementation of $\mathcal{F}^{(\mathrm{Xtn})}$ (tolerating all corruption patterns).

We can prove a composition theorem for this notion of security. For a protocol $\pi=\pi[\mathcal{G}]$ and a protocol $\gamma$ we use $\pi[\gamma / \mathcal{G}]$ to denote the protocol $\pi$ where the use of $\mathcal{G}$ has been replaced by $\gamma$. Let $\mathrm{Xtn}^{\pi}\left(\mathrm{Xtn}^{\gamma}\right)$ be the extensions $\pi(\gamma)$ associates to its ideal functionalities. For $\mathcal{H} \in \pi[\gamma / \mathcal{G}]$ we associate the extension $\mathrm{Xtn}(\mathcal{H})=\mathrm{Xtn}^{\pi}(\mathcal{H})$ when $\mathcal{H} \in \pi$ and $\mathrm{Xtn}(\mathcal{H})=\mathrm{Xtn}^{\gamma}(\mathcal{H})$ when $\mathcal{H} \in \gamma$.

Theorem 8. Assume that $\pi$ is an Xtn-secure implementation of $\mathcal{F}$ and $\mathcal{G} \in \pi$ with $\mathrm{Xtn}^{\pi}(\mathcal{G})=\mathrm{Xtn}_{\mathcal{G}}$. Assume furthermore that $\gamma$ is an $\mathrm{Xtn}_{\mathcal{G}}$-secure implementation of $\mathcal{G}$. Then $\pi[\gamma / \mathcal{G}]$ is an Xtn-secure implementation of $\mathcal{F}$.

Proof. When $\mathrm{Xtn}^{\pi}(\mathcal{G})=\mathrm{Xtn}_{\mathcal{G}}$ for $\mathcal{G} \in \pi$, then $\pi$ being an Xtn-secure implementation of $\mathcal{F}$ implies that $\widehat{\pi}\left[\mathcal{G}^{\left(\mathrm{Xtn}_{\mathcal{G}}\right)}\right]$ is a UC secure implementation of $\mathcal{F}^{(\mathrm{Xtn})}$. That $\gamma$ is an $\mathrm{Xtn}_{\mathcal{G}}$-secure implementation of $\mathcal{G}$ implies that $\widehat{\gamma}$ is a UC secure implementation of $\mathcal{G}^{\left(\mathrm{Xtn}_{\mathcal{G}}\right)}$. So, by the UC composition theorem, $\widehat{\pi}\left[\widehat{\gamma} / \mathcal{G}^{\left(\mathrm{Xtn}_{\mathcal{G}}\right)}\right]$ is a UC secure implementation of $\mathcal{F}^{(\mathrm{Xtn})}$. Since $\left.\widehat{\pi[\gamma / \mathcal{G}}\right]=\widehat{\pi}\left[\widehat{\gamma} / \mathcal{G}^{\left(\mathrm{Xtn}_{\mathcal{G}}\right)}\right]$ this implies that $\widehat{\pi[\gamma / \mathcal{G}]}$ is a UC secure implementation of $\mathcal{F}^{(\mathrm{Xtn})}$ which by definition implies that $\pi[\gamma / \mathcal{G}]$ is an Xtn-secure implementation of $\mathcal{F}$.

\subsection{Asymmetric Trust}

We now use Definition [5 to express asymmetric trust, formalizing the concepts we introduced in Section 1.4. To each $p_{i}$ we associate an adversary structure $\mathcal{A}_{i}$ expressing that $p_{i}$ trusts that only corruption patterns PAT $\in \mathcal{A}_{i}$ will actually occur. We call $\underline{\mathcal{A}}=\left(\mathcal{A}_{1}, \ldots, \mathcal{A}_{n}\right)$ an aggregate adversary structure. A symmetric adversary structure $\mathcal{A}$ corresponds to the aggregate adversary structure $\underline{\mathcal{A}}^{n}=(\mathcal{A}, \ldots, \mathcal{A})$. For an actually occurring corruption pattern PAT $=$ 
(Act, Pas) we let Foreseeing $\mathcal{A}_{\mathcal{A}}(\mathrm{PAT})=\left\{p_{i} \in \mathcal{P} \backslash\right.$ Pas $\left.\mid \mathrm{Pat} \in \mathcal{A}_{i}\right\}$ and we let $\mathrm{NAÏVE}_{\underline{\mathcal{A}}}(\mathrm{PAT})=\left\{p_{i} \in \mathcal{P} \backslash \mathrm{PAS} \mid \mathrm{PAT} \notin \mathcal{A}_{i}\right\}$. We call $p_{i} \in$ ForeSEEING $_{\underline{\mathcal{A}}}(\mathrm{PAT})$ foreseeing and we call $p_{i} \in \mathrm{NAÏVE}_{\underline{\mathcal{A}}}(\mathrm{PAT})$ naïve. We model asymmetric trust by treating the foreseeing honest parties as we normally treat the honest parties in UC security and treating the corrupted parties as we do normally. For the naïve parties we allow the simulator (environment) extra powers, using the concepts we defined earlier. Formally, we say that a corruption extension Xtn is an extension for $\underline{\mathcal{A}}=\left(\mathcal{A}_{1}, \ldots, \mathcal{A}_{n}\right)$ if it holds for all PAT that $\mathrm{Xtn}(\mathrm{PAT})=$ (Actin, ActOut, Pasin, PasOut) satisfies PasIn, PasOut, Actin, ActOut $\subseteq \mathrm{NAÏVE}_{\mathcal{A}}(\mathrm{PAT})$. This gives a lot of granularity in how to treat the honest-butnaïve parties: PASIN specifies the naïve parties for which the inputs are allowed to leak to the adversary, PASOUT specifies the naïve parties for which the outputs are allowed to leak to the adversary, AстIn specifies the naïve parties for which the inputs might be controlled by the adversary, and AстOUT specifies the naïve parties for which the outputs might by controlled be the adversary.

To get some more structure, we name some special types of extensions, called relaxed, semi-relaxed, strong, strict, which are defined as follows:

- Xtn is of type relaxed if it is the extension of $\underline{\mathcal{A}}$ that specifies ACTIN $=$ ACTOUT $=\mathrm{NAÏVE}_{\underline{\mathcal{A}}}(\mathrm{PAT})$ for all PAT, i.e., there is no security for naïve parties.

- Xtn is of type semi-relaxed if it is the extension of $\underline{\mathcal{A}}$ that specifies ACTIN $=$ $\emptyset$, ACtOut $=$ NAÏVE $\backslash$ ACT, PASIN $=$ NAÏVE $\backslash$ PAS, PasOut $=$ NAÏVE $\backslash$ PAS. I.e., the honest-but-naïve parties are guaranteed that their inputs are contributed correctly to the computation. They are however not guaranteed to receive correct outputs nor any privacy of their inputs or their outputs.

- Xtn is of type strong if it is the extension of $\underline{\mathcal{A}}$ that specifies ACTIN $=$ ActOut $=\emptyset$, PASIN $=$ PASOUt $=\operatorname{NAÏVE}_{\underline{\mathcal{A}}}(\mathrm{PAT})$, i.e., naïve parties have no privacy but may contribute their inputs and get correct results.

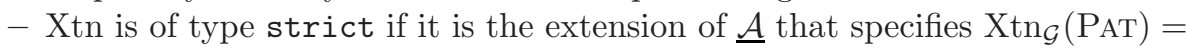
$(\emptyset, \emptyset, \emptyset, \emptyset)$, i.e., there is full security for naïve parties.

If ATK is one of relaxed, semi-relaxed, strong, strict, we call $\pi$ an ATK $\underline{\mathcal{A}}$-secure implementation of $\mathcal{F}$ if $\pi$ is an Xtn-secure implementation of $\mathcal{F}$ tolerating $\underline{\mathcal{A}}$, where Xtn is the extension of $\underline{\mathcal{A}}$ of type ATK. Also, if $\pi$ makes use of functionality $\mathcal{G}$, we say that $\mathcal{G}$ is an ATK functionality if the extension $\pi$ assigns to $\mathcal{G}$ is of type ATK.

The following composition theorem is an immediate corollary to Theorem 8 ,

Corollary 1. Let ATK, $\mathrm{ATK}^{\prime} \in\{$ relaxed, semi-relaxed, strong, strict $\}$. If $\pi$ is an ATK $\underline{\mathcal{A}}$-secure implementation of $\mathcal{F}$, where $\mathcal{G} \in \pi$ is an $\mathrm{ATK}^{\prime}$ functionality, and $\gamma$ is an ATK $^{\prime} \underline{\mathcal{A}}$-secure implementation of $\mathcal{G}$, then $\pi[\gamma / \mathcal{G}]$ is an ATK $\underline{\mathcal{A}}$-secure implementation of $\mathcal{F}$.

Note that the notion of semi-relaxed security as defined here is equivalent to the notions sender-independent validity and dealer-independent correctness in Section 2. Indeed, defining broadcast and VSS by requiring a semi-relaxed secure 
implementation of a corresponding ideal functionality would define exactly these notions.

To see the connection between symmetric security and our notions of asymmetric security, let $\underline{\mathcal{A}}$ be the aggregate adversary structure modeling that all parties trust that at most $t$ parties will be corrupted, and let $\pi$ be a protocol using only strict functionalities. In this case the simulator is given no extra corruption when at most $t$ parties are corrupted, as all parties are foreseeing. So, as long as at most $t$ parties are corrupted, relaxed, semi-relaxed, strong and strict $\underline{\mathcal{A}}$-security are equivalent to the usual UC $t$-security. If however more than $t$ parties are corrupted, then all honest parties are naïve, meaning e.g. that strong security allows the simulator to see the inputs and outputs of all parties, and relaxed security allows the simulator to specify the inputs and outputs of all parties. So, when more than $t$ parties are corrupted, strong $\underline{\mathcal{A}}$-security gives no guarantees on the privacy of any party but still guarantees correctness for the honest-but-naïve parties, and relaxed $\underline{\mathcal{A}}$-security gives no guarantees at all. Note that giving no guarantees at all when more than $t$ parties are corrupted is equivalent to normal $t$-security, where simulation is only required for environments corrupting at most $t$ parties. Therefore relaxed security is a generalization of normal (symmetric) UC security, and strong security is a strengthening.

\section{Multi-Party Computation in the UC Framework}

In this section we first formalize the notion of secure multi-party computation in the UC framework where the parties have asymmetric trust in each other. In Section 2.3 we already informally looked at this case in the secure-channels model. In Section 4.2 we look at a setting where a number of certificate authorities (or common reference strings) are present and where the parties have asymmetric trust in these certificate authorities (or common reference strings).

\subsection{Secure Function Evaluation}

For simplicity we focus on secure function evaluation (SFE). SFE of $f\left(x_{1}, \ldots, x_{n}\right)$ can be expressed as securely evaluating the ideal functionality $\mathcal{F}_{\text {SFE }}^{f}$ for secure function evaluation of $f$. Essentially $\mathcal{F}_{\mathrm{SFE}}^{f}$ takes an input $x_{i}$ from each $p_{i}$, computes $\left(y_{1}, \ldots, y_{n}\right)=f\left(x_{1}, \ldots, x_{n}\right)$ and outputs $y_{i}$ securely to $p_{i}$. We call $\pi$ an $\underline{\mathcal{A}}$ SFE $\mathrm{w} /$ full relaxation of $f$ if $\pi$ is a relaxed $\underline{\mathcal{A}}$-secure implementation of $\overline{\mathcal{F}}_{\mathrm{SFE}}^{f}$. We call $\pi$ an $\underline{\mathcal{A}}$ SFE w/ contributor-independent correctness of $f$ if $\pi$ is a semi-relaxed $\underline{\mathcal{A}}$-secure implementation of $\mathcal{F}_{\mathrm{SFE}}^{f}$. For concreteness we flesh out these notions below.

Definition 6 (SFE w/ full relaxation). The simulator has the following extra powers:

INPUT SECRECY: If $p_{i}$ is honest and $F \notin \mathcal{A}_{i}$ or $p_{i}$ is corrupt, then the simulator sees $x_{i}$. If party $p_{i}$ is honest and $F \in \mathcal{A}_{i}$ then the simulator is not shown $x_{i}$. 
InPut CoRrectness: If $p_{i}$ is honest and $F \notin \mathcal{A}_{i}$ or $p_{i}$ is corrupt, then the simulator can replace $x_{i}$ by some $x_{i}^{\prime}$. After this, the outputs $\left(y_{1}, \ldots, y_{n}\right)=$ $f\left(x_{1}^{\prime}, \ldots, x_{n}^{\prime}\right)$ are computed, where $x_{i}^{\prime}=x_{i}$ for all honest $p_{i}$ with $F \in \mathcal{A}_{i}$.

OutPut SECRECY: If $p_{i}$ is honest and $F \notin \mathcal{A}_{i}$ or $p_{i}$ is corrupt, then the simulator sees $y_{i}$. If party $p_{i}$ is honest and $F \in \mathcal{A}_{i}$ then the simulator is not shown $y_{i}$.

Output Correctness: If $p_{i}$ is honest and $F \notin \mathcal{A}_{i}$ or $p_{i}$ is corrupt, then the simulator can replace $y_{i}$ by some $y_{i}^{\prime}$. After this, $\mathcal{F}_{\mathrm{SFE}}^{f}$ outputs $y_{i}^{\prime}$ on behalf of $p_{i}$, where $y_{i}^{\prime}=y_{i}$ for all honest $p_{i}$ with $F \in \mathcal{A}_{i}$.

RoBustness: Robustness is best expressed as a condition on the protocol (as opposed to the simulation), by requiring that all honest parties compute an output, i.e., no honest party aborts the protocol. Alternatively, one can require this only for the foreseeing parties, getting weak robustness.

Definition 7 (SFE w/ contributor-independent correctness). The simulator has the following extra powers (listing only differences from Definition [6):

InPUT CORRECTNESS: If $p_{i}$ is corrupt, then the simulator can replace $x_{i}$ by some $x_{i}^{\prime}$. After this, the outputs $\left(y_{1}, \ldots, y_{n}\right)=f\left(x_{1}^{\prime}, \ldots, x_{n}^{\prime}\right)$ are computed, where $x_{i}^{\prime}=x_{i}$ for all honest $p_{i}$.

These notions can be generalized to MPC w/ full relaxation and MPC w/ contributor-independent correctness by requiring a relaxed (semi-relaxed) $\underline{\mathcal{A}}-$ secure implementation of a more general ideal functionality $\mathcal{F}$.

\subsection{With Asymmetrically Trusted Setup}

We now consider a setting where some setup is given. We focus on UC security, where setup is needed when there is no trust among the parties. We consider two setup assumptions which have been studied previously: common reference string (CRS) and key registration (KR), and we generalize the study to consider asymmetric trust. Here, we only cover the KR case whereas the CRS case is treated in the full version of the paper, using similar techniques.

Key Registration. In 1 Barak et al. gave a feasibility result for UC secure MPC in a network which had a key registration service $\mathcal{F}_{\mathrm{KR}}$ which allows a user $U_{i}$ to register a public key $p k_{i}$ while checking that $U_{i}$ knows a corresponding secret key. We extend this analysis of the power of key registration by analyzing a setting where there are several key registration services (KRS's) in which the users have different partial trust. For completeness we also assume that the users have different, partial trust in each other. We characterize the aggregate adversary structures which allow to securely compute any ideal functionality in this setting. We consider the same type of security as in [16]: polynomial time security and the protocol is only required to deliver outputs if all parties are honest. This is modeled by allowing the simulator to decide when and if honest

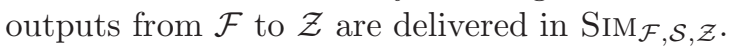


We model the KRS's as parties $\mathcal{K} \mathcal{R}=\left\{K R_{k}\right\}$. We then add $n$ users $\mathcal{U}=$ $\left\{U_{1}, \ldots, U_{n}\right\}$, making the party set $\mathcal{P}=\mathcal{K} \mathcal{R} \cup \mathcal{U}$. The users are the parties which want to compute some ideal functionality $\mathcal{F}_{\mathcal{U}}$ among them 4 We also add a strict functionality for authenticated, asynchronous point-to-point communication among the users and between the users and the KRS's, and we add a strict functionality for secure, asynchronous point-to-point communication among the users 5 Finally we need that each user can give a proof of possession (PoP) of the secret key when it registers a public key. For this purpose we postulate an ideal functionality $\mathcal{P O} \mathcal{P}$. Let gen be the generator $p k=\operatorname{gen}(r)$ used to generate public keys (we can wlog assume that the randomness $r$ constitutes the private key). We assume that $\mathcal{P O P}$ behaves as follows: On input $\left(U_{i}, K R_{k}, r_{i}\right)$ from $U_{i}$, output $\left(U_{i}, K R_{k}, p k_{i}=\operatorname{gen}\left(r_{i}\right)\right)$ to $K R_{k}$. This models that $U_{i}$ gives $p k_{i}$ to $K R_{k}$ and then somehow proves knowledge of $r_{i}$ such that $p k_{i}=\operatorname{gen}\left(r_{i}\right)$.

By $U_{i}$ registering a public key at $K R_{k}$ we then mean that $U_{i}$ samples a random public key $p k_{i} \leftarrow \operatorname{gen}\left(r_{i}\right)$ and inputs $\left(U_{i}, K R_{k}, r_{i}\right)$ securely to $\mathcal{P O} \mathcal{P}$. The honest behavior of each $K R_{k}$ is as follows: The first time it sees $\mathcal{P O} \mathcal{P}$ output $\left(U_{i}, K R_{k}, p k_{i}\right)$ for $U_{i}$ it sends $\left(U_{i}, K R_{k}, p k_{i}\right)$ to all users $U_{j}$ using authenticated point-to-point communication.

Since the behavior of $K R_{k}$ is fixed and the behavior of $\mathcal{P O P}$ is given by gen, we specify a protocol by $\pi=\left(\right.$ gen $\left., U_{1}, \ldots, U_{n}\right)$. For convenience we assume that each $U_{i}$ starts the protocol by registering some $p k_{i, k}$ with each $K R_{k}$. Then $U_{i}$ waits for each $K R_{k}$ to send some $p k_{j, k}$ for each $U_{j} \in \mathcal{U}$ and stores all these keys. After this registration phase the users then proceed to run the actual protocol. We can therefore in the specification of $p_{i}$ assume that it knows the keys $p k_{k, j}$. We call such a $\pi=\left(\right.$ gen, $\left.U_{1}, \ldots, U_{n}\right)$ a KR-protocol.

As for trust, we consider only active corruptions, so that PAT $=(\mathrm{ACT}, \mathrm{ACT})$ for all patterns. We therefore write $\mathrm{ACT} \in \mathcal{A}$ and consider $\mathcal{A} \in 2^{\mathcal{P}}$. We associate no trust to the KRS's. That is, we assume that $\mathcal{A}_{K R_{k}}=2^{\mathcal{P}}$ for each KRS. To each $U_{i}$ we associate a corruption structure $\mathcal{A}_{i} \subseteq 2^{\mathcal{P}}$. We call $\left(\mathcal{A}_{1}, \ldots, \mathcal{A}_{n}\right)$ complete for the $\mathrm{KR}$ setting if it allows to securely compute any efficient ideal functionality $\mathcal{F}_{\mathcal{U}}$ among the users using a $\mathrm{KR}$ protocol.

For $\operatorname{ACT}_{i} \in \mathcal{A}_{i}$ we let $\operatorname{ACT}_{i}^{\mathcal{U}}=\operatorname{ACT}_{i} \cap \mathcal{U}$ and $\operatorname{ACT}_{i}^{\mathcal{K} \mathcal{R}}=\operatorname{ACT}_{i} \cap \mathcal{K} \mathcal{R}$. We say that two users $U_{i} \neq U_{j}$ are $\mathrm{KR}$ connected if it holds for all $\mathrm{ACT}_{i} \in \mathcal{A}_{i}$ and $\mathrm{ACT}_{j} \in \mathcal{A}_{j}$ that either $\mathrm{ACT}_{i}^{\mathcal{K} \mathcal{R}} \neq \mathcal{K} \mathcal{R}$ or $\operatorname{ACT}_{j}^{\mathcal{K} \mathcal{R}} \neq \mathcal{K} \mathcal{R}$ or $\operatorname{ACT}_{i}^{\mathcal{U}} \cup \operatorname{ACT}_{j}^{\mathcal{U}} \neq \mathcal{U}$. That is, together, $U_{i}$ and $U_{j}$ cannot imagine a scenario where both of them think that all KRS's might be corrupted and where together they think all users might be corrupted.

\footnotetext{
${ }^{4}$ We say that $\mathcal{F}_{\mathcal{P}^{\prime}}$ is among $\mathcal{P}^{\prime}$ if it ignores $p_{i}$-inputs for $\mathcal{P} \backslash \mathcal{P}^{\prime}$ and gives no $p_{i}$-outputs for $\mathcal{P} \backslash \mathcal{P}^{\prime}$.

${ }^{5}$ Since secure, asynchronous point-to-point communication has a normal UC secure implementation given authenticated channels and several standard complexity assumptions, this strict ideal functionality can be replaced with any such implementation to get an equivalent model with only authenticated communication, using Corollary 1
} 
Theorem 9. An aggregate adversary structure $\underline{\mathcal{A}}=\left(\mathcal{A}_{1}, \ldots, \mathcal{A}_{n}\right)$ is complete for the KR setting iff all pairs of distinct users are $K R$ connected in $\underline{\mathcal{A}}$.

The proof of Theorem 9 is given in the full version of the paper. A special case of Theorem 9 is when the users have no trust in each other $\left(\mathcal{A}_{i}^{\mathcal{U}}=\mathcal{U}\right.$ for all $\left.U_{i}\right)$ in which case the condition can be phrased as: There exists at most one user who thinks that all KRS's can be corrupted.

\section{Conclusion}

We proposed a notion of asymmetric trust in protocol security and gave a general definition of asymmetric secure MPC and gave specialized definitions of asymmetric secure broadcast, VSS, and SFE. We explored the feasibility of broadcast, VSS, and MPC in various models with asymmetric trust. A tight characterization of the feasibility of broadcast has been found for asymmetric trust, and nontrivial upper and lower bounds for VSS, and we have shown how to tolerate strictly stronger adversaries in MPC than with symmetric trust. It is an open problem to completely characterize the aggregate adversary structures that allow for MPC in the case with active adversaries and no set-up.

\section{References}

1. Barak, B., Canetti, R., Nielsen, J.B., Pass, R.: Universally composable protocols with relaxed set-up assumptions. In: 45th Annual Symposium on Foundations of Computer Science, pp. 186-195. IEEE, Los Alamitos (2004)

2. Baum-Waidner, B., Pfitzmann, B., Waidner, M.: Unconditional Byzantine agreement with good majority. In: 8th Annual Symposium on Theoretical Aspects of Computer Science. LNCS, vol. 480, Springer, Heidelberg (1991)

3. Ben-Or, M., Goldwasser, S., Wigderson, A.: Completeness theorems for noncryptographic fault-tolerant distributed computation. In: 20th ACM Symposium on the Theory of Computing, pp. 1-10 (1988)

4. Blakley, G.R.: Safeguarding cryptographic keys. In: 1979 National Computer Conference. AFIPS Conference proceedings, vol. 48, AFIPS Press (1979)

5. Canetti, R.: Universally composable security: A new paradigm for cryptographic protocols. In: 42nd Annual Symposium on Foundations of Computer Science, pp. 136-145 (2001) Full version in IACR ePrint Archive 2000/067

6. Canetti, R., Lindell, Y., Ostrovsky, R., Sahai, A.: Universally composable twoparty and multi-party secure computation. In: Proceedings of the Thirty-Fourth Annual ACM Symposium on the Theory of Computing, pp. 494-503 (2002)

7. Chor, B., Goldwasser, S., Micali, S., Awerbuch, B.: Verifiable secret sharing and achieving simultaneity in the presence of faults. In: 26th IEEE Symposium on the Foundations of Computer Science, pp. 383-395 (1985)

8. Cleve, R.: Limits on the security of coin flips when half the processors are faulty (extended abstract). In: 18th ACM Symposium on the Theory of Computing, pp. 364-369 (1986)

9. Cramer, R., Damgård, I., Dziembowski, S., Hirt, M., Rabin, T.: Efficient multiparty computations secure against an adaptive adversary. In: Stern, J. (ed.) EUROCRYPT 1999. LNCS, vol. 1592, Springer, Heidelberg (1999) 
10. Fitzi, M., Gisin, N., Maurer, U., von Rotz, O.: Unconditional Byzantine agreement and multi-party computation secure against dishonest minorities from scratch. In: Knudsen, L.R. (ed.) EUROCRYPT 2002. LNCS, vol. 2332, pp. 482-501. Springer, Heidelberg (2002)

11. Fitzi, M., Gottesman, D., Hirt, M., Holenstein, T., Smith, A.: Detectable Byzantine agreement secure against faulty majorities. In: 21st ACM Symposium on Principles of Distributed Computing (PODC), pp. 118-126 (2002)

12. Goldreich, O., Micali, S., Wigderson, A.: How to play any mental game - a completeness theorem for protocols with honest majority. In: 19th ACM Symposium on the Theory of Computing, pp. 218-229 (1987)

13. Hirt, M., Maurer, U.: Player simulation and general adversary structures in perfect multiparty computation. Journal of Cryptology 13(1), 31-60 (2000)

14. Maurer, U.: Secure multi-party computation made simple. In: Cimato, S., Galdi, C., Persiano, G. (eds.) SCN 2002. LNCS, vol. 2576, pp. 14-28. Springer, Heidelberg (2003)

15. Maurer, U.: Towards a theory of consistency primitives. In: Guerraoui, R. (ed.) DISC 2004. LNCS, vol. 3274, pp. 379-389. Springer, Heidelberg (2004)

16. Pease, M., Shostak, R., Lamport, L.: Reaching agreement in the presence of faults. Journal of the ACM 27(2), 228-234 (1980)

17. Pfitzmann, B., Waidner, M.: Information-theoretic pseudosignatures and Byzantine agreement for $t>=n / 3$. Technical Report RZ 2882 (\#90830), IBM Research (1996)

18. Rabin, T., Ben-Or, M.: Verifiable secret sharing and multiparty protocols with honest majority. In: 21st ACM Symposium on the Theory of Computing, pp. 73$85(1989)$

19. Shamir, A.: How to share a secret. CACM 22, 612-613 (1979)

20. Yao, A.C.: Protocols for secure computations. In: 23rd IEEE Symposium on the Foundations of Computer Science, pp. 160-164. IEEE, Los Alamitos (1982) 